\title{
Impact of using a non-diabetes-specific risk calculator on eligibility for statin therapy in type 2 diabetes
}

\author{
H. C. Price • R. L. Coleman • R. J. Stevens • \\ R. R. Holman
}

Received: 28 October 2008 /Accepted: 30 October 2008 /Published online: 2 December 2008

(C) Springer-Verlag 2008

\begin{abstract}
Aims/hypothesis The aim of this study was to investigate the impact of using a non-diabetes-specific cardiovascular disease (CVD) risk calculator to determine eligibility for statin therapy according to current UK National Institute for Health and Clinical Excellence (NICE) guidelines for those patients with type 2 diabetes who are at an increased risk of CVD (10 year risk $\geq 20 \%)$.

Methods The 10 year CVD risks were estimated using the UK Prospective Diabetes Study (UKPDS) Risk Engine and the Framingham equation for 4,025 patients enrolled in the Lipids in Diabetes Study who had established type 2 diabetes and LDL-cholesterol $<4.1 \mathrm{mmol} / \mathrm{l}$.

Results The mean (SD) age of the patients was 60.7 (8.6) years, blood pressure $141 / 83(17 / 10) \mathrm{mmHg}$ and the total cholesterol:HDL-cholesterol ratio was 3.9 (1.0). The median (interquartile range) diabetes duration was $6(3-11)$ years and the $\mathrm{HbA}_{1 \mathrm{c}}$ level was $8.0 \%$ (7.2-9.0\%). The cohort comprised $65 \%$ men, $91 \%$ whites, $4 \%$ Afro-Caribbeans, 5\% Asian Indians and $15 \%$ current smokers. More patients were classified as being at high risk by the UKPDS Risk Engine $(65 \%)$ than by the Framingham CVD equation $(63 \%)$ $(p<0.0001)$. The Framingham CVD equation classified fewer men and people aged $<50$ years old as high risk $(p<0.0001)$. There was no difference between the UKPDS
\end{abstract}

H. C. Price $(\bowtie) \cdot R$. L. Coleman · R. R. Holman

Diabetes Trials Unit, Oxford Centre for Diabetes,

Endocrinology and Metabolism, University of Oxford,

Churchill Hospital,

Oxford OX3 7LJ, UK

e-mail: Hermione.Price@dtu.ox.ac.uk

R. J. Stevens

Cancer Research UK Epidemiology Unit, University of Oxford,

Oxford, UK
Risk Engine and Framingham classification of women at high risk $(p=0.834)$.

Conclusions/interpretation These results suggest that the use of Framingham-derived rather than UKPDS Risk Engine-derived CVD risk estimates would deny about one in 25 patients statin therapy when applying current NICE guidelines. Thus, under these guidelines the choice of CVD risk calculator is important when assessing CVD risk in patients with type 2 diabetes, particularly for the identification of the relatively small proportion of younger people who require statin therapy.

Keywords Clinical science - Macrovascular disease . Mathematical modelling and simulation

\begin{tabular}{|c|c|}
\hline \multicolumn{2}{|c|}{ Abbreviations } \\
\hline CVD & cardiovascular disease \\
\hline NICE & $\begin{array}{l}\text { National Institute for Health and Clinical } \\
\text { Excellence }\end{array}$ \\
\hline UKPDS & UK Prospective Diabetes Study \\
\hline LDS & Lipids in Diabetes Study \\
\hline SCORE & Systematic Coronary Risk Evaluation \\
\hline DECODE & $\begin{array}{l}\text { Diabetes Epidemiology: Collaborative analysis } \\
\text { of Diagnostic Criteria in Europe }\end{array}$ \\
\hline ARDS & Collaborative Atorvastatin Diabetes Study \\
\hline
\end{tabular}

\section{Introduction}

Cardiovascular disease (CVD) is a major problem for people with type 2 diabetes, who are at substantially greater risk of CVD compared with the general population even when age, ethnicity, income, cholesterol level, systolic blood pressure and smoking are taken into account [1]. The 
relative impact of lowering blood pressure and LDLcholesterol is similar in individuals with and without diabetes, but the higher absolute risk in those with diabetes means such treatment results in greater absolute risk reductions.

In the UK, prescription of statin therapy is regulated by National Institute for Health and Clinical Excellence (NICE) guidelines, which allow the use of statins in adults with clinical evidence of CVD or for primary prevention in adults with an estimated 10 year CVD risk of $\geq 20 \%$ [2]. NICE defines CVD as disease of the heart and blood vessels [2]. The choice of CVD risk calculators includes the Framingham risk equation [3] and the UK Prospective Diabetes Study (UKPDS) Risk Engine [4], but the Framingham risk equation and other non-diabetes-specific risk calculators have been shown to underestimate CVD risk in diabetic cohorts [5]. One reason for this could be that only 428 individuals with diabetes were followed in the Framingham study [3]. Also, the Framingham risk equation includes diabetes only as a dichotomous risk factor (present or absent), along with traditional CVD risk factors [3]. The UKPDS Risk Engine, by contrast, is a type 2 diabetes-specific risk calculator that uses both a continuous measure of glycaemia $\left(\mathrm{HbA}_{1 \mathrm{c}}\right)$ and the duration of known diabetes, in addition to traditional CVD risk factors [4]. Unlike the Framingham equation, it can be used in AfroCaribbean and Asian-Indian populations as well as white populations.

The present study was designed to compare the performance of the UKPDS Risk Engine and the recently updated (2008) Framingham CVD risk equation [3] for the determination of the proportion of UK individuals with type 2 diabetes thought to be eligible for statin therapy according to current NICE guidelines [2] using data from a representative sample of the population. In addition, we investigated the use of a risk calculator to determine if younger people are at high enough CVD risk to warrant statin therapy.

\section{Methods}

Participants We used baseline (pre-randomisation) data for 4,025 of the 4,191 participants recruited into the Lipids in Diabetes Study (LDS) between 1999 and 2001 for whom the requisite data were available. This population was chosen as being likely to be representative of communitybased patients with type 2 diabetes who are possibly eligible for primary CVD prevention therapies. LDS was a lipid-lowering study involving 30 UK centres, in which patients were enrolled on the basis that their need for lipid lowering was uncertain given an absence of known coronary heart disease and an inclusion criterion requiring LDL-cholesterol levels to be $<4.1 \mathrm{mmol} / \mathrm{l}$. To compare the relative performance of the two calculators in younger people, a subset of 552 individuals aged between 40 and 50 years were examined.

Methods The 10 year CVD risks were estimated for each individual using the UKPDS Risk Engine (version 3) [4] and the 2008 Framingham CVD risk equation [3]. The UKPDS Risk Engine defines CVD as fatal or non-fatal myocardial infarction, ischaemic heart disease, sudden cardiac death, fatal or non-fatal stroke or death following peripheral vascular disease. The Framingham equation defines CVD as fatal or non-fatal myocardial infarction, angina pectoris, coronary insufficiency or fatal or non-fatal stroke, transient ischaemic attack, peripheral vascular disease (intermittent claudication) or heart failure. The conventional risk factors used by both calculators are age, sex, smoking status, total and HDL-cholesterol and systolic blood pressure. Only the UKPDS Risk Engine includes atrial fibrillation. The CVD risk estimates calculated using the two methods were compared using the sign test. The proportion of men, women and participants aged $<50$ years who were not eligible for statin therapy were compared using the McNemar test.

\section{Results}

Demographics The mean (SD) age of the patients was 60.7 (8.6) years, blood pressure was $141 / 83(17 / 10) \mathrm{mmHg}$ and the total cholesterol:HDL-cholesterol ratio was 3.9 (1.0). The median (interquartile range) diabetes duration was 6 (3-11) years and $\mathrm{HbA}_{1 \mathrm{c}}$ was $8.0 \%$ (7.2-9.0\%). The cohort comprised $65 \%$ men, $91 \%$ whites, $4 \%$ Afro-Caribbeans, $5 \%$ Asian Indians and 15\% current smokers. In addition, $0.47 \%$ had atrial fibrillation.

Distribution of 10 year CVD risk estimates The proportion of patients with an estimated 10 year CVD risk of $\geq 20 \%$ was $2 \%$ lower $(p<0.0001)$ using the Framingham risk equation (63\%) compared with the UKPDS Risk Engine (65\%) (Fig. 1). The corresponding proportions for men only were $78 \%$ vs $82 \%(p<0.0001)$, for women only were $34 \%$ vs $34 \%(p=0.834)$ and for individuals $<50$ years of age were $11 \%$ vs $18 \%(p<0.0001)$. The proportion of this population with an estimated 10 year CVD risk of $\geq 10 \%$ was $93 \%$ using the UKPDS Risk Engine and 90\% using the Framingham risk equation.

\section{Discussion}

This study found small but significant differences in the proportion of people identified as being at an increased risk 


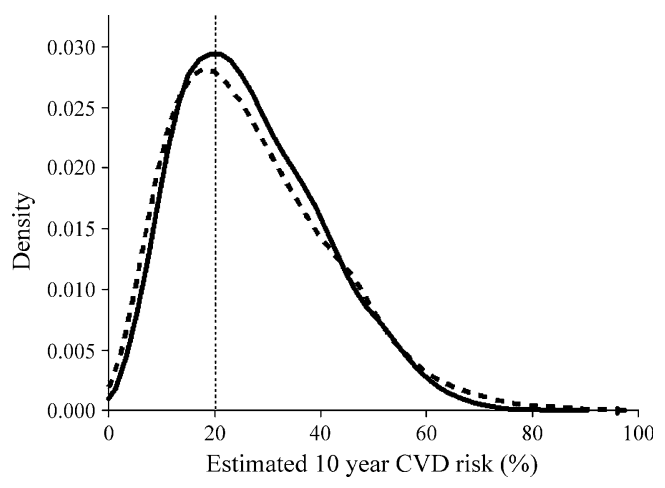

Fig. 1 Kernel density plot showing the distribution of the estimated 10 year CVD risk calculated using the Framingham equation (broken line) and the UKPDS Risk Engine (solid line). The dotted line at 20\% indicates the threshold value for statin therapy eligibility under current NICE guidelines. A greater proportion of patients were classified as being at high risk by the UKPDS Risk Engine (65\%) than by the Framingham CVD equation $(63 \%)(p<0.0001)$

of CVD, depending on the risk calculator used. When the Framingham equation rather than the UKPDS Risk Engine was used, $2 \%$ less of the total study cohort, $4 \%$ less men and $7 \%$ less individuals aged $<50$ years were estimated to have a 10 year CVD risk of $\geq 20 \%$. This would result in these individuals being denied statin therapy according to current UK NICE guidelines.

LDS participants were chosen as they represent a population in whom, under current guidelines, there may be some uncertainty about initiating statin therapy. They had no previous CVD events and as such were a relatively low-risk population. Their mean $\mathrm{HbA}_{1 \mathrm{c}}$ was, however, $1 \%$ higher than that reported by more recent studies on individuals with type 2 diabetes managed in the community [6]. Compared with other sources of UK primary care data, the measurements for the LDS population indicated that participants included in this study tended to be younger and had a longer duration of diabetes [7]. As well as population changes in $\mathrm{HbA}_{1 \mathrm{c}}$ and blood pressure, death from CVD has also declined in men (but not women) with diabetes over the last 30 years, possibly as a result of improvements in lifestyle and the treatment of CVD [8]. The vast majority of participants included in these analyses were white, but the ethnic mix of the cohort was not dissimilar to that of the UK as a whole at the time of the study [9]. Although this may mean that the absolute proportion of this population entitled to statin therapy may have fallen, it should not alter the relative performance of the two risk calculators.

The UKPDS Risk Engine (version 3) and the 2008 Framingham CVD equation were compared because of their similarities in the definition of CVD and because versions of both have been validated in their respective populations, although the most recent Framingham CVD equation has yet to be validated. The Framingham equation has previously been shown to underperform in diabetic populations [5], and this may in part be because it includes diabetes as a dichotomous variable rather than a continuous measure of glycaemia. UKPDS Risk Engine CVD risk estimates decrease by approximately $15-20 \%$ for each $1 \%$ reduction in $\mathrm{HbA}_{1 \mathrm{c}}$.

No long-term LDS outcome data are available to compare CVD risk estimates with observed events, as the LDS trial was terminated early following the withdrawal of cerivastatin for safety reasons [10]. The 2008 Framingham 10 year CVD risk equation estimates the proportion of individuals eligible for statin therapy to be similar to that estimated by the UKPDS Risk Engine. However, the updated Framingham equation requires information on antihypertensive treatment, which was not available for the LDS cohort. The numbers shown here assume that the blood pressure measurements in LDS participants at baseline were untreated; this is likely to be the case given the mean blood pressure was $141 / 83$ and recruitment took place between 1999 and 2001. If everyone in the study was receiving blood pressure therapy, the 10 year estimated risk of CVD would increase significantly to $77 \%$. The Framingham and UKPDS definitions of CVD are similar, with the exception that the Framingham equation includes individuals with angina pectoris (brief recurrent chest discomfort for up to $15 \mathrm{~min}$, brought on by exertion and relieved by rest or glyceryl trinitrate) [11], whereas the UKPDS Risk Engine includes those with ischaemic heart disease (based on ECG Minnesota coding) [12]. Because of the broader definition of CVD used by the Framingham equation, it would be expected that it would tend to classify more individuals as being at high CVD risk, but this is not the case.

The differences in 10 year CVD risk estimates are seen predominantly in men and those $<50$ years of age. The difference in estimated risk in men may be due to the failure of the Framingham risk equation to show a gradient of risk when total cholesterol in men is $<6.2 \mathrm{mmol} / 1$ [13]. Studies have demonstrated a continuous relationship between total cholesterol and CVD risk. In the LDS, the mean (SD) total cholesterol level was $4.8(0.8) \mathrm{mmol} / \mathrm{l}$. The Framingham risk equation has also been found to underestimate risk when risk factors are present in severe forms (very elevated systolic blood pressure or total cholesterol) [13]. These factors may explain the difference in male risk estimates found in this study.

In the UKPDS, 71 individuals aged $<50$ years had a coronary heart disease event. However, in the Framingham study no one under the age of 52 years had a CVD event [14]. This difference in event rates underlying the two equations is likely to explain the underestimation of risk in younger people by the Framingham equation. 
European guidelines recommend statin therapy for any individual with type 2 diabetes who are aged $>40$ years [15]. Although we were not able to investigate individuals $\leq 40$ years of age, we did look at individuals aged $<50$ years, and have shown that the Framingham equation classifies fewer individuals as being at high CVD risk than does the UKPDS Risk Engine $(p<0.0001)$. These data illustrate the value of using a diabetes-specific risk calculator to identify the relatively small number of younger individuals requiring statin therapy. If the NICE guidelines were to be reviewed and the cut-off value for statin therapy lowered to include all individuals with an estimated 10 year CVD risk of $\geq 10 \%$, this would result in the vast majority (93\%) of individuals with type 2 diabetes being entitled to statin therapy.

Until NICE guidance changes to this $\geq 10 \%$ level, we recommend using a diabetes-specific risk calculator. Once NICE guidelines change it is likely that a risk calculator will be unnecessary to determine eligibility for statin therapy except when it is being considered for an individual aged $\leq 40$ years.

Given that statins have now been found to be safe and efficacious even in children and adolescents [16], future work will simulate lifetimes to determine the optimum age or level of risk at which initiation of statin therapy should be considered in individuals with type 2 diabetes. We are also currently examining the ability of the two risk equations to correctly identify incident cardiovascular disease in the European Prospective Investigation of Cancer (EPIC)-Norfolk population [17].

\section{Conclusion}

While the current UK NICE guidelines are enforced the choice of CVD risk calculator can be important when assessing CVD risk in patients with type 2 diabetes, particularly for the identification of younger people at high CVD risk. If NICE guidelines change to recommend statin therapy for primary prevention in all individuals with a 10 year CVD risk of $\geq 10 \%$, then the vast majority of people with type 2 diabetes will be entitled to statin therapy, irrespective of the risk calculator chosen.

Acknowledgements H. C. Price is funded by a Diabetes Trials Unit Fellowship.

Duality of interest The authors declare that there is no duality of interest associated with this manuscript.

\section{References}

1. Stamler J, Vaccaro O, Neaton J, Wentworth D (1993) Diabetes, other risk factors, and 12-yr cardiovascular mortality for men screened in the multiple risk factor intervention trial. Diabetes Care 16:434-444

2. National Institute for Health and Clinical Excellence (2006) Statins for the prevention of cardiovascular disease. NICE, London. Available from http://www.nice.org.uk/TA094, accessed 7 November 2008

3. D'Agostino R, Vasan R, Pencina M et al (2008) General cardiovascular risk profile for use in primary care: The Framingham Heart Study. Circulation 117:743-753

4. Coleman R, Stevens R, Holman R (2007) Estimating cardiovascular disease risk for individuals with type 2 diabetes: the new UKPDS Risk Engine. Diabetic Medicine 24(Suppl 1):56 (Abstract)

5. Coleman R, Stevens R, Retnakaran R, Holman R (2007) Framingham, SCORE, and DECODE risk equations do not provide reliable cardiovascular risk estimates in type 2 diabetes. Diabetes Care 30:1292-1293

6. Asimakopoulou K, Skinner T, Spimpolo J, Marsh S, Fox C (2008) Unrealistic pessimism about risk of coronary heart disease and stroke in patients with type 2 diabetes. Patient Educ Couns 71:95-101

7. Farmer A, Wade A, Goyder E et al (2007) Impact of self monitoring of blood glucose in the management of patients with non-insulin treated diabetes: open parallel group randomised trial. BMJ 335:132

8. Gregg E, Qiuping G, Cheng Y, Venkat-Narayan K, Cowie C (2007) Mortality trends in men and women with diabetes, 1971 to 2000. Ann Intern Med 147:149-155

9. http://www.statistics.gov.uk/cci/nugget.asp?id=273, accessed 26 August 2008

10. Maggin M, Raschetti R, Traversa G et al (2004) The cerivastatin withdrawal crisis: a "post-mortem" analysis. Health Policy 69:151157

11. Lloyd-Jones DM, Larson MG, Beiser A, Levy D (1999) Lifetime risk of developing coronary heart disease. Lancet 353:89-92

12. UK Prospective Diabetes Study Group (1991) UK Prospective Diabetes Study (UKPDS). VIII. Study design, progress and performance. Diabetologia 34:877-890

13. Grundy S, Pasternak R, Greenland P, Smith S, Fuster V (1999) AHA/ACC Scientific Statement. Assessment of cardiovascular risk by use of multiple-risk-factor assessment equations: a statement for healthcare professionals from the American Heart Association and the American College of Cardiology. Circulation 100:1481-1492

14. Hiller R, Sperduto R, Podgor M, Ferris F, Wilson P (1988) Diabetic retinopathy and cardiovascular disease in type II diabetics. The Framingham Heart Study and the Framingham Eye Study. Am J Epidemiol 128:402-409

15. De Backer G, Ambrosioni E, Borch-Johnsen K et al (2003) European guidelines on cardiovascular disease prevention in clinical practice. Third Joint Task Force of European and Other Societies on Cardiovascular Disease Prevention in Clinical Practice. Eur Heart J 24:1601-1610

16. McCrindle B, Ose L, Marais A (2003) Efficacy and safety of atorvastatin in children and adolescents with familial hypercholesterolemia or severe hyperlipidemia: a multicenter, randomized, placebo-controlled trial. J Pediatrics 143:74-80

17. Day N, Oakes S, Luben R et al (1999) EPIC-Norfolk: study design and characteristics of the cohort. European Prospective Investigation of Cancer. Br J Cancer 80(Suppl 1):95-103 